\title{
Curl-B technique applied to $S$ warm constellation for determining field-aligned currents
}

\author{
Patricia Ritter and Hermann Lühr \\ GFZ Potsdam, Telegrafenberg, D-14473 Potsdam, Germany \\ (Received November 15, 2004; Revised May 30, 2005; Accepted June 1, 2005; Online published April 14, 2006)

\begin{abstract}
The constellation of the Swarm satellites provides for the first time the opportunity to determine field-aligned currents in the ionosphere uniquely. This is achieved by employing the curl-B relation of Ampere's law directly to measurements of a satellite pair flying side-by-side. The new technique is applied to a set of consistent magnetic field and current data generated by a global magnetospheric model. Using a realistic Swarm constellation the current distribution is determined along the orbit from the synthetic magnetic field data. The resulting currents are tested against the input currents. The agreement between input model and recovered field-aligned currents is excellent and much improved compared to the single-satellite estimates. Due to the spatial separation of the sampling points, only the distribution of large-scale field-aligned currents can be determined. These investigations demonstrate one important aspect of the broad capabilities provided by the upcoming space mission.
\end{abstract}

Key words: External magnetic field, high latitude currents, field-aligned currents, current determination.

\section{Introduction}

Field-aligned currents (FACs) are of paramount importance for high-latitude ionospheric physics since they carry the energy and momentum from the magnetosphere into the upper atmosphere. Their spatial distribution and temporal variation in the polar regions have been studied increasingly since spaceborne magnetic field vector data became available (Zmuda et al., 1966). The first complete maps of the statistical location of Birkeland currents (FACs) in the polar region were developed by Zmuda and Armstrong (1974) and by Iijima and Potemra (1976). In addition to the space physics aspects it is important for further improvements of the main field and lithospheric field modeling to understand and reduce the influence of magnetospheric and ionospheric currents.

The methods used to determine polar FAC density, $\mathbf{j}$, from magnetic field satellite data, B, are all based on Ampere's law:

$$
\operatorname{curl} \mathbf{B}=\mu_{0} \mathbf{j}
$$

Recently, the following techniques are in use:

The gradient $\left(d B_{y} / d x\right)$ method is used quite commonly and estimates the local FAC density from the along-track difference of the transverse magnetic component on a trackby-track basis (e.g. Fung and Hoffman, 1992; Lühr et al., 1996; Stauning et al., 2001). For this method it is generally assumed that the currents are constant over the time span of passage and that the FACs are organized in sheets. The current sheet assumption, however, does not account for the 2D spatial distributions and filamentary structures of the FACs

Copyright (c) The Society of Geomagnetism and Earth, Planetary and Space Sciences (SGEPSS); The Seismological Society of Japan; The Volcanological Society of Japan; The Geodetic Society of Japan; The Japanese Society for Planetary Sciences; TERRAPUB in the auroral regions and therefore provides an incomplete image of the complex ionospheric current system. To gain a more global picture, the horizontal magnetic field deflections of many polar passes for the same IMF conditions may be interpolated bivariately. The curl of this continuous magnetic perturbation field yields the spatial distribution of the FACs in the polar region (Stauning, 2002). This method involves an averaging of the signatures over time and space, and it is obvious that neighbouring structures of up and downward directed FACs will be diminished or even cancel each other. This may generally result in too small current densities. In addition, the temporal evolution of the FAC system which may follow an IMF sector change cannot be accounted for by this technique. Stauning et al. (2005) give an overview of most of these techniques employed for single-satellite data and their limitations.

To overcome these problems, a large number of satellites would be needed. A first attempt to recover the full time/space structure of the FAC distribution is made by utilising the magnetic field readings of the 66 satellites of the Iridium System (Anderson et al., 2000; Waters et al., 2001). The limiting factor in this case, however, is the poor sampling of the magnetic field. The magnetometers have a resolution of $48 \mathrm{nT}$, and instantaneous readings are transmitted every 200s. This corresponds to a sampling distance of about $16^{\circ}$ of latitude. Special data fitting techniques are required to get meaningful results out of such a highly undersampled data set (Waters et al., 2001). The measurements are reported to be most useful for determining the FAC distribution during higher level activity.

Field-aligned currents are known to vary strongly not only in time, but also in space, according to the prevailing solar wind (SW) conditions. A further problem is the geometry of the assumed current sheet orientation. Several publications offer ideas how to incorporate the angle be- 
tween the flight direction and the sheet direction (e.g. Fung and Hoffman, 1992; Lühr et al., 1996). A useful tool for finding the orientation of the current layer is the minimum variance analysis (Sonnerup and Cahill, 1967). In reality, however, the current sheets are neither infinite nor planar, so all solutions offered are just approximations. Any deviation of the current geometry from the infinite sheet results in an underestimation of the current intensity. To obtain a more reliable picture of the FAC structure across the polar regions nearly simultaneous overflights of two satellites (e.g. CHAMP and Ørsted) have been considered (Vennerstrøm et al., 2002). Unfortunately, such coincidences are quite rare. Since Ampere's law requires the spatial derivatives of the transverse magnetic field components in both horizontal directions for the unambiguous estimation of FACs, all single satellite techniques fall short of the simultaneous cross-track gradient. Many of these problems can be solved with data from two satellites flying side-by-side.

Apart from considering the geometry of the FACs one has to keep in mind that the magnetic field measured by a satellite contains contributions from many sources. Naturally, only those parts of the field that are caused by FACs should be accounted for in the estimation. This can be achieved by applying the Helmholtz theorem whereby, in general, any vector field (here B) can be decomposed in a scalar, $V$, and a vector potential, $\mathbf{A}$

$$
\mathbf{B}=\operatorname{grad} V+\operatorname{curl} \mathbf{A}
$$

When applying the curl operator to this vector field the contribution from the scalar potential vanishes since curl $\operatorname{grad} V=0$. For this reason all contributions of sources below and above the orbital plane are suppressed. Only magnetic fields generated by currents crossing the orbital plane contribute to the second term on the righthand side of Eq. (2).

Given the case that spatially distributed magnetic field observations are available, as is provided by the Swarm constellation, the application of Ampere's law for determining FACs ensures that potential fields will not influence the result. If, however, the FACs are determined from single satellite data where only the along-track gradient of the field is considered, residual potential fields can affect the resulting current densities.

The multi-satellite mission Swarm is conceived to investigate the dynamics of the Earth's magnetic field and its interaction with the Earth system in unprecedented detail (Friis-Christensen et al., 2006). The instrumentation on board will provide among others high precision vector data of the electric and magnetic fields. With the planned constellation of three satellites in near-polar orbits (inclination $\approx 87^{\circ}$ ) at two different heights, one at $530 \mathrm{~km}$ and a pair at initially $450 \mathrm{~km}$, the mission is particularly well suited to study the complex current systems of the polar ionosphere. The lower pair shall fly side-by-side, separated in this study by only $1.6^{\circ}$ in longitude which is equivalent to $\approx 180$ kilometres in east/west direction at the equator. The simultaneous, longitudinally spaced measurements provide the possibility to include the missing cross-track component directly in the computation and produce more complete results. This allows for the first time to determine field-aligned currents in the ionosphere unambiguously by directly employing the curl-B relation.

In order to study the advantages and possibilities offered by the Swarm constellation a synthetic dataset of magnetic and electric field components, as well as ionospheric currents, was produced by the Danish Space Research Institute in collaboration with the Community Coordinated Modeling Center (CCMC) of the Goddard Space Flight Center (Vennerstrøm et al., 2004; Vennerstrom et al., 2006; Moretto et al., 2006). A global Magneto-Hydro-Dynamics (MHD) model was used to simulate the interaction of the solar wind with the magnetosphere. The resulting fieldaligned currents were closed in the ionosphere. By employing an empirical model for the ionospheric conductivity the spatial distribution of the electric potential was deduced, and Hall and Pedersen currents in the ionosphere could be computed. From the latter the 3D distribution of magnetic field deflections was derived. The magnetic and electric field components were computed on a spherical grid extending from $90 \mathrm{~km}$ to $4 \mathrm{RE}$. The simulations were performed for varying IMF orientations representing quiet to moderately disturbed geomagnetic conditions. In this MHD model the activity is controlled by the amplitude of IMF $B_{Z}$. This comprehensive 3D database of magnetic field components and ionospheric currents was used to test our ideas for the new technique to compute field-aligned currents.

In this paper we present the new method developed for the Swarm mission (Section 2). The application and test against the input model currents is given in Section 3. In Section 4 we compare the results with single satellite estimates, followed by a discussion of the quality and an assessment of the application to real data in Section 5.

\section{Determination of the Field-Aligned Currents}

The basic equation for determining the electric current density, $\mathbf{j}$, from magnetic field observations, $\mathbf{B}$, is Ampere's law. In the auroral region the only currents expected to flow at satellite altitude $(\sim 400 \mathrm{~km})$ are the field-aligned currents. Therefore only one component of curl $\mathbf{B}$ is of importance

$$
j_{z}=\frac{1}{\mu_{0}}\left(\frac{\partial B_{y}}{\partial x}-\frac{\partial B_{x}}{\partial y}\right)
$$

where $x, y, z$ are components of a local field-aligned Cartesian coordinate system (MFA, see Appendix).

As can be seen from Eq. (3), the spatial gradients are needed for deriving the current. The Swarm constellation with its closely spaced lower satellite pair SwA and SwB provides a suitable set-up for this purpose. As shown in Fig. 1, the two satellites are separated in east-west direction. Flying side-by-side they provide the cross-track gradient of $B_{x}$, while the difference of readings taken at two points in time $\left(t_{1}\right.$ and $\left.t_{2}\right)$ are used as the along-track gradient of $B_{y}$. The combination of these gradients according to Eq. (3) allows to determine the average current density, $j_{z}$, that flows through the trapezoid (see Fig. 1). The result is assigned locally to the centre of the four measurement points.

For practical reasons, and for a better visualisation of the processing steps required, the curl-B operation is done in a locally horizontal Cartesian coordinate system called 


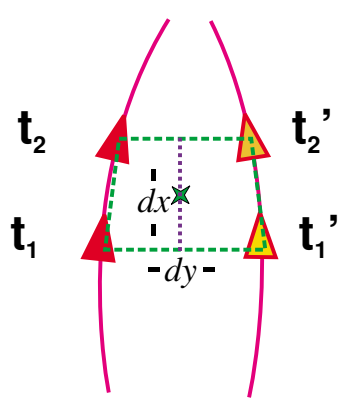

SwA

ascending

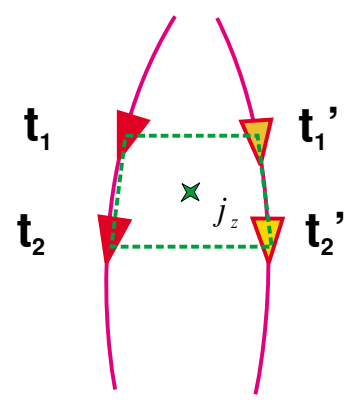

SwA

descending
Fig. 1. Schematic drawing of the orbit configuration for determining the radial currents.

Velocity-Zenith (VZ) frame (see Appendix). The origin is the midpoint between the four sampling positions shown in Fig. 1. For this set-up spacecraft SwA is selected as the reference satellite. Due to a small lead or lag in orbital phase of the second satellite (SwB), the corresponding readings are taken from SwB at a time t', when the positions of the two spacecraft form a symmetric quad. The $x$ component of the VZ system is defined by the horizontal component of the mean velocity vector of the two spacecraft, $z$ points radially outward and the $y$ component completes the triad.

It should be noted that in this computation process, the vertical current component is determined as $j_{z}$ in the VZ frame, rather than the field-aligned component, $j_{\|}$. The recovery of the FAC density from the vertical component, however, is straight-forward:

$$
j_{\|}=\frac{j_{z}}{\sin I}
$$

where $I$ is the inclination of the magnetic field. This simple relation holds since all currents at satellite altitude are confined to the field-aligned direction. Subsequently, we will deal only with the radial component, $j_{z}$, as a measure for the FAC density. As a further advantage this current component can be compared directly with the radial component, $j_{r}$, of the input model.

Since there are 4 measurement points available for the calculations of the gradients, the mean value of each difference pair goes into the estimation of the curl in Eq. (3):

$$
j_{z}=\frac{1}{\mu_{0}}\left(\frac{d B_{y}(\mathrm{~A})+d B_{y}(\mathrm{~B})}{d x(\mathrm{~A})+d x(\mathrm{~B})}-\frac{d B_{x}\left(\mathrm{t}_{1}\right)+d B_{x}\left(\mathrm{t}_{2}\right)}{d y\left(\mathrm{t}_{1}\right)+d y\left(\mathrm{t}_{2}\right)}\right)(5)
$$

Differences denoted e.g. (A) are differences taken at satellite SwA between times $t_{1}$ and $t_{2}$. Differences denoted e.g. $\left(t_{1}\right)$ are differences taken at time $\left(t_{1}\right)$ between measurements at SwA and SwB. To avoid problems with very small distances $|d y|$ at the cross-over points of the orbits near the poles, cross-track differences around the points of closest approach are omitted.

Apart from the method shown above there is also the possibility to determine the field-aligned current density from Ampere's integral law

$$
\mathbf{j}=\frac{1}{\mu_{0} A} \oint \mathbf{B} \cdot \mathbf{d} \mathbf{l}
$$

where $\mathbf{d l}$ is a path element along the integration way. The integration has to be performed along a closed path and $A$ is the encircled area (in our case the trapezoid). If we apply Eq. (6) to discrete measurements, as sketched in Fig. 1, the equation for calculating the vertical current density, $j_{z}$, takes the following form:

$$
\begin{aligned}
j_{z}=\frac{2}{\mu_{0}}[ & \left(B_{v A}\left(\mathrm{t}_{1}\right)+B_{v A}\left(\mathrm{t}_{2}\right)\right) d \ell_{v A} \\
& +\left(B_{t A}\left(\mathrm{t}_{2}\right)+B_{t B}\left(\mathrm{t}_{2}^{\prime}\right)\right) d \ell_{t_{2}} \\
& -\left(B_{v B}\left(\mathrm{t}_{2}^{\prime}\right)+B_{v B}\left(\mathrm{t}_{1}^{\prime}\right)\right) d \ell_{v B} \\
& \left.-\left(B_{t B}\left(\mathrm{t}_{1}\right)+B_{t A}\left(\mathrm{t}_{1}\right)\right) d \ell_{t_{1}}\right] \\
& \cdot \frac{1}{\left(d \ell_{v A}+d \ell_{v B}\right)\left(d \ell_{t_{1}}+d \ell_{t_{2}}\right)}
\end{aligned}
$$

where, $B_{v A}$ and $B_{v B}$ are the magnetic field components parallel to the flight direction of SwA and SwB, respectively, and $B_{t A}$ and $B_{t B}$ are the components aligned with the connecting line between the accompanying spacecraft. In this case, $B_{v}$ and $B_{t}$ are not orthogonal. $d \ell_{v}$ and $d \ell_{t}$ are the along-track and transverse path elements, respectively. Again, numerical problems are expected at the cross-over points, where $d \ell_{t}$ becomes very small. In this study we made no use of Ampere's integral for determining the FAC density, as it yields practically the same results as the curl-B described before in Eq. (5).

\section{Application to Synthetic Data}

For the development and test of the method described above we use the three-dimensional grid of synthetic magnetic field components resulting from the global MHD simulation runs of the near-Earth plasma dynamics (for details see Vennerstrøm et al., 2004; Vennerstrom et al., 2006; Moretto et al., 2006). The underlying models represent the present state of the art in solar wind-magnetosphere coupling. The B-field of the synthetic data set is given in a spherical centred-dipole coordinate system, where the longitude is related to local time (Solar-Magnetic (SM) frame, see Appendix). This frame is suited for presenting the ionospheric electro-dynamics. The synthetic magnetic field data are given on a grid spaced by $\Delta \theta=1.4^{\circ}, \Delta \phi=2.8^{\circ}$ in latitude and longitude, respectively. The griding in radial direction, $\Delta r$, assumes values ranging from $1 \mathrm{~km}$ to $50 \mathrm{~km}$ (increasing spacing) with $r$ reaching from $90 \mathrm{~km}$ to $700 \mathrm{~km}$. For the determination of the expected measurements we interpolate the synthetic magnetic components linearly between the grid points along the orbits of the Swarm satellites to obtain a continuous, equidistant sampling with $\Delta t=5 \mathrm{~s}$. The orbit data (position and velocity) for the Swarm spacecraft were computed as part of the Endto-End Mission Simulator Study (Olsen et al., 2004). These position and velocity data are given in an Earth-fixed system ITRF (see Appendix). For practical reasons it was decided to do all current calculations in the SM frame. Therefore the ephemerides are transformed into this dipole system using a location of the dipole axis at $78.68^{\circ}$ latitude and $289.6^{\circ}$ lon- 

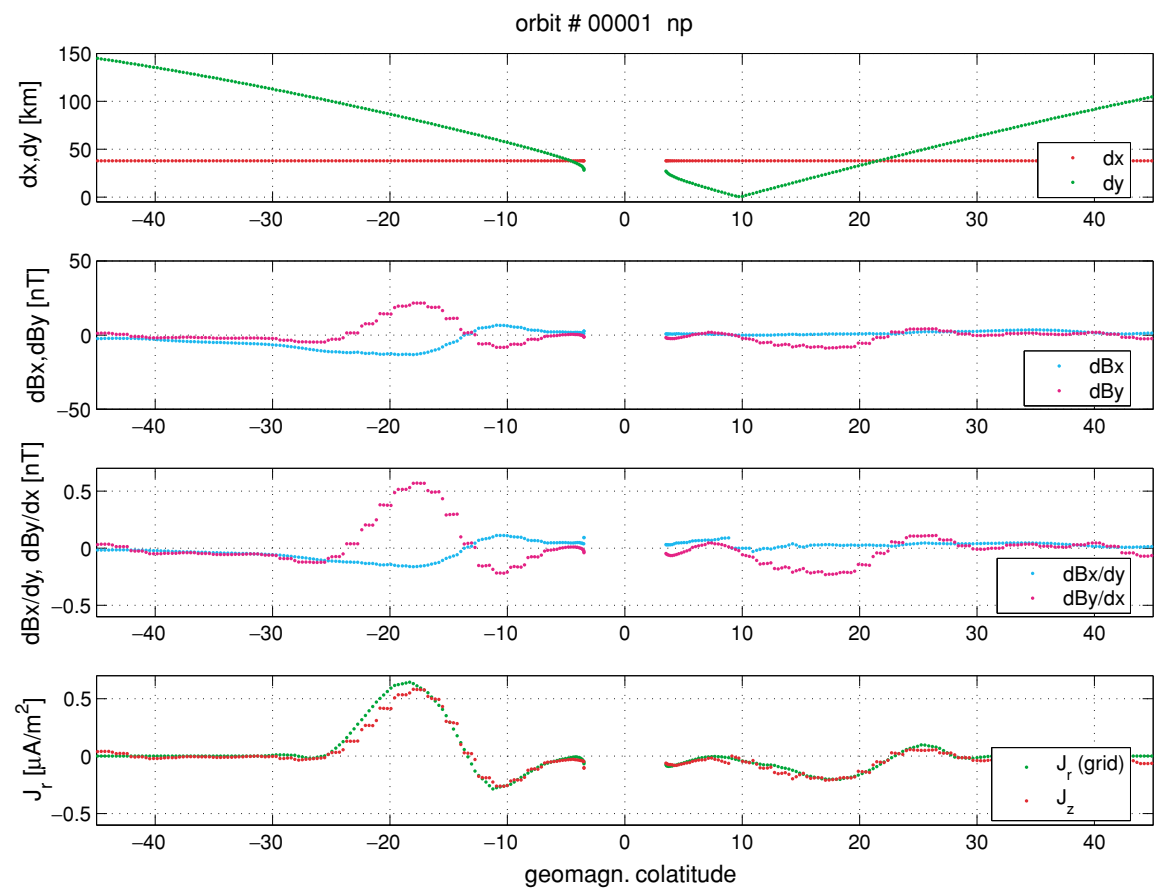

Fig. 2. Variation of the different terms in Eq. (5) across the pole (IMF $B_{Z}=-2.5 \mathrm{nT}$, for details see text).

gitude in geographic coordinates. These values are derived from the dipole terms of a recent magnetic field model (e.g. Holme et al., 2003).

The magnetic field given in the synthetic data set is the disturbance field caused by the currents. No geomagnetic main field has been added to it. Thus it can be used directly for the current retrieval. The B-field is given in spherical components $\left(B_{r}, B_{\theta}, B_{\phi}\right)$ in the $\mathrm{SM}$ frame. For our purposes, the horizontal components $\left(B_{\theta}, B_{\phi}\right)$ at both spacecraft are rotated locally about the radial axis into the VZ frame giving $\left(B_{x}, B_{y}\right)$. This is done in two steps. First, the $B_{\theta}$ component is rotated into the flight direction using the angle $\alpha$, which is calculated individually at the four sampling points

$$
\begin{array}{ll}
\alpha=\sin ^{-1}\left(\frac{\sin \theta_{m}}{\sin \theta_{j}}\right) & \text { for descending tracks } \\
\alpha=180^{\circ}-\alpha & \text { for ascending tracks }
\end{array}
$$

$\theta_{m}$ is the co-latitude of the orbit's closest approach to the pole and $\theta_{j}$ are the co-latitudes of the sampling points. In the second step, the horizontal components are rotated further by half of the orbit separation angle, $\pm 0.8^{\circ}$, for SwA and $\mathrm{SwB}$, respectively, to align them with the $x$ and $y$ axes.

For the execution of Eq. (5) we need to employ the spatial derivatives of the magnetic field. The along-track gradient of the transverse magnetic field, $d B_{y}$, is derived from successive readings that are $5 \mathrm{~s}$ apart. The cross-track gradient of the field component, $d B_{x}$, is obtained from the difference of corresponding readings of the two spacecraft. The separation between these points varies in this example from about $100 \mathrm{~km}$ at $30^{\circ}$ colatitude to zero at the crossover points (see Fig. 2). While the separation of the measurement points in cross-track direction, $d_{y}$, is given by the orbital geometry. We tested several spacings in the along- track direction. The $5 \mathrm{~s}$, giving a distance of about $38 \mathrm{~km}$ for $d_{x}$, turned out to be a good compromise. When using larger separations an undesirable damping of the current signature resulted. Smaller steps did not yield any improvement. In the vicinity of the crossover points the baseline for deriving reliable cross-track gradients becomes too small. We introduced a lower limit for $d_{y}$ (here $3 \mathrm{~km}$ ), below which the second term in Eq. (5) is set to zero. This means, the current determination is incomplete in a time interval of $\pm 15 \mathrm{~s}$ around the singular point. Fortunately, the largest contribution comes from the along-track $\Delta B_{y}$ term, therefore the results in this interval are still usable.

Figure 2 shows the variations of the different terms in Eq. (5) and their contributions to the retrieved radial current density for one pass over the north pole. The various quantities are plotted versus magnetic colatitude, where negative angles represent the ascending and positive angles denote the descending parts of the track. The apparent data gaps in the middle of each frame reflect the fact that the orbits do not cross the magnetic pole. In the top panel we display the distances between the measurement points. The cross-track separation of the spacecraft is getting smaller as the two spacecraft move towards the pole and, in this case, $d_{y}$ passes the cross-over point at $10^{\circ}$ off the magnetic pole. In the second panel we show the magnetic field differences in the two directions. The along-track gradient of the $B_{y}$ component does not vary smoothly but step-like. This is a consequence of the linear interpolation process that was used to retrieve the magnetic field samples from the grid points at the orbit positions. In the third panel the along-track and cross-track gradients are presented. It is quite evident that the largest contribution to the radial current estimate comes from the along-track gradient of the $B_{y}$ component. As mentioned before, the cross-track gradient was set to zero in the vicinity of the cross-over point $(d y<3 \mathrm{~km})$. 

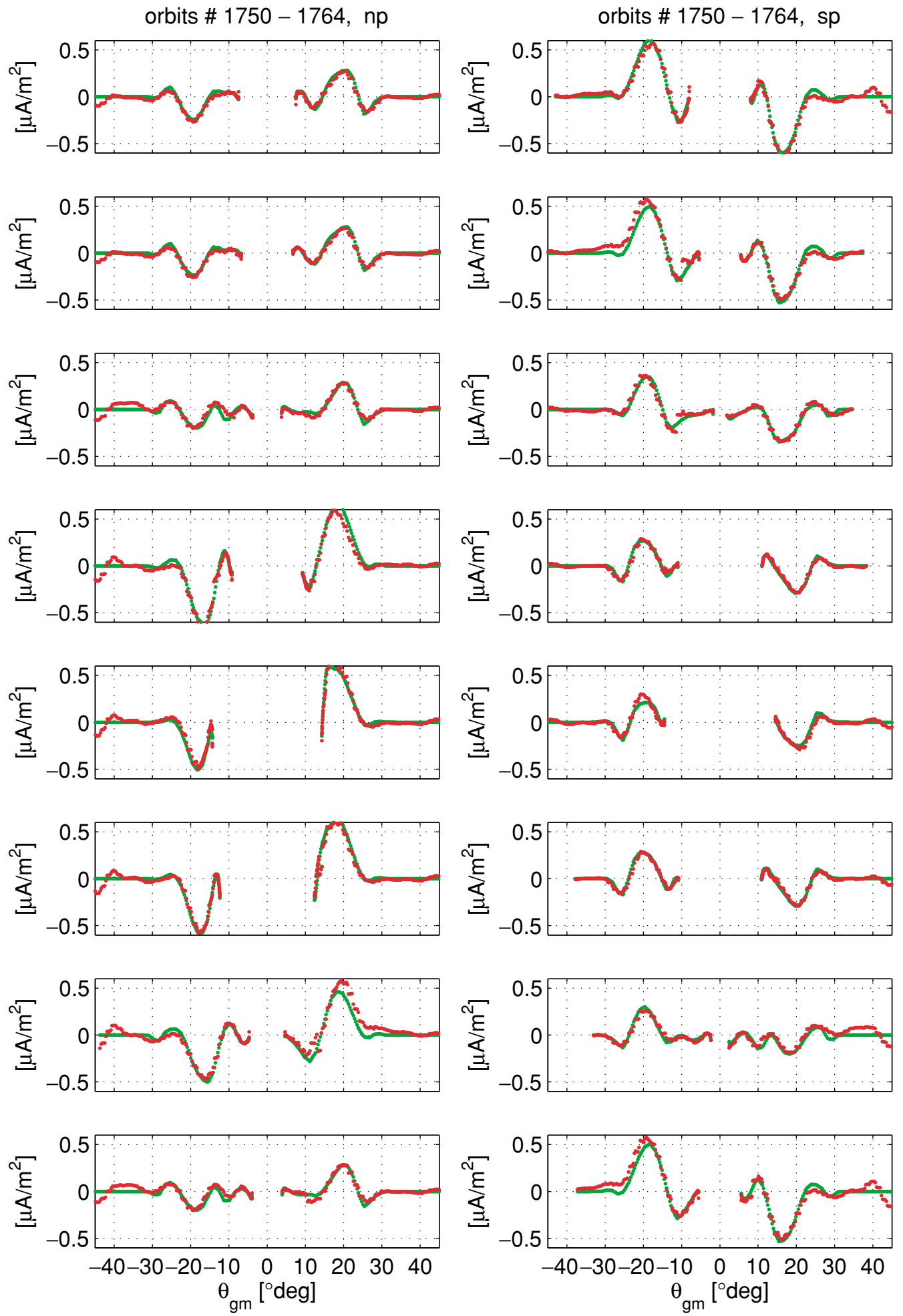

$$
\mathrm{j}_{\mathrm{r}} \text { (grid) }
$$

Fig. 3. Direct comparison of derived radial current density (red) and the corresponding model input data (green) (IMF $B_{Z}=-2.5 \mathrm{nT}$ ) for the northern (np) and southern (sp) hemispheres.

The bottom panel contains the comparison of the derived radial current density, $j_{z}$, with the synthetic input values of the radial current component, $j_{r}$. In principle there is a good agreement between these two current values. Nevertheless, we want to point out that there seem to be systematic dis- crepancies (a small phase shift) between the two curves. Estimated current features appear closer to the pole.

After going through the individual processing steps of estimating the radial current component along one polar crossing, we now have a look at a larger number of passes. 

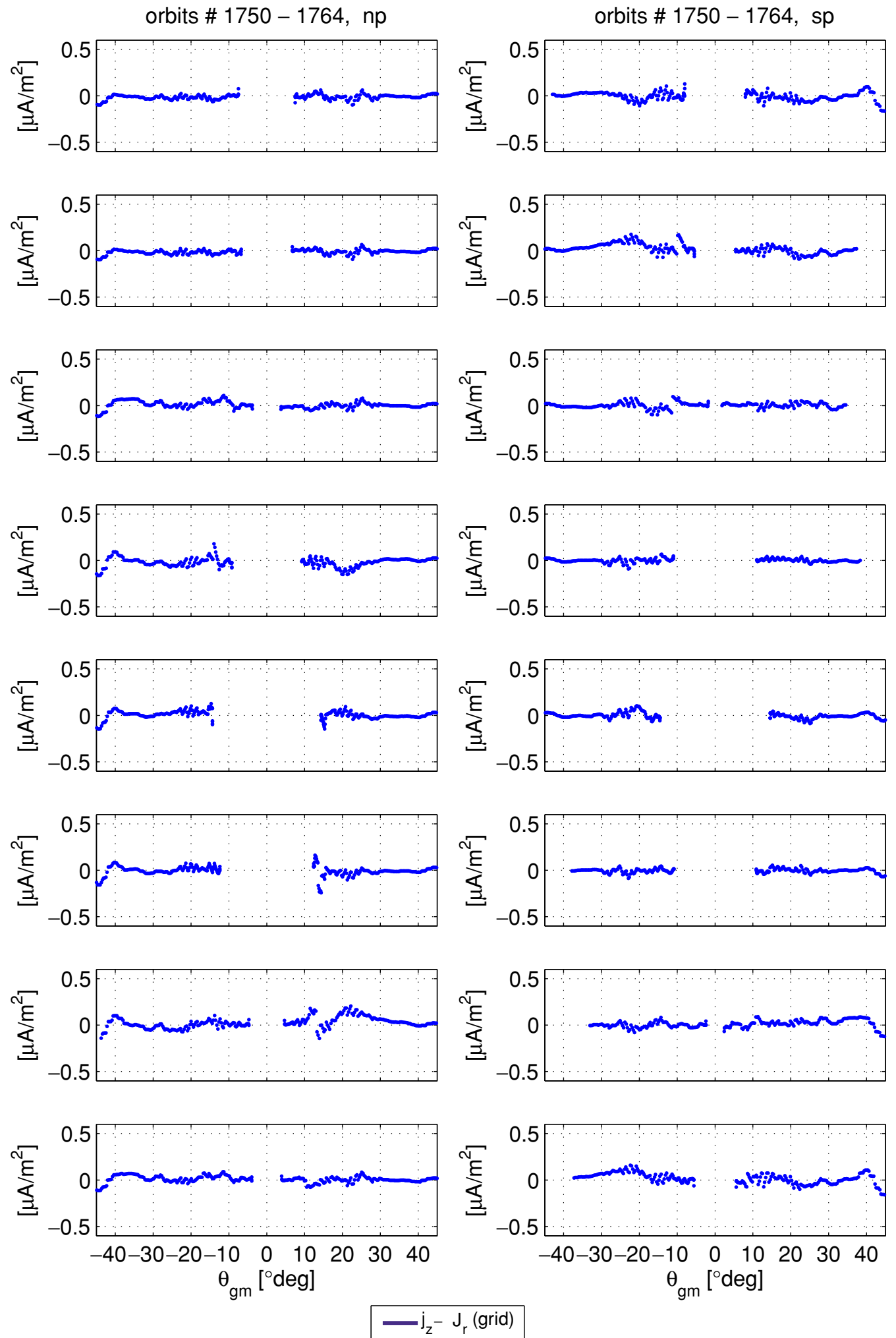

Fig. 4. Difference between the derived radial current density and the corresponding model input data (IMF $B_{Z}=-2.5 \mathrm{nT}$ ).

Figure 3 shows every second polar pass of one day for both the northern and southern hemisphere. For all these cases we find an almost perfect agreement between the derived and input current densities in both hemispheres.

For a more quantitative view of the deviations we have plotted the differences between the derived and modelled currents in Fig. 4 for the same tracks. The prominent fea- tures to be noted are: the enhanced scatter in some places due to the gridding of the B-field, the sinusoidal variations caused by the phase shift, and some discontinuous jumps in the vicinity of the cross-over points (e.g. second and third frame in the right column, near $-10^{\circ}$ ). All these deflections are at least one order of magnitude smaller than the current amplitudes. 


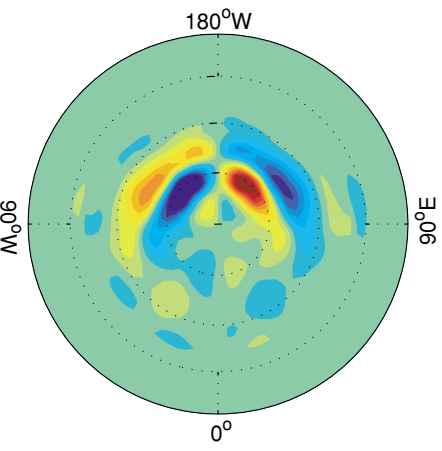

$J_{r}\left(\right.$ grid) $\left[\mu \mathrm{A} / \mathrm{m}^{2}\right]$

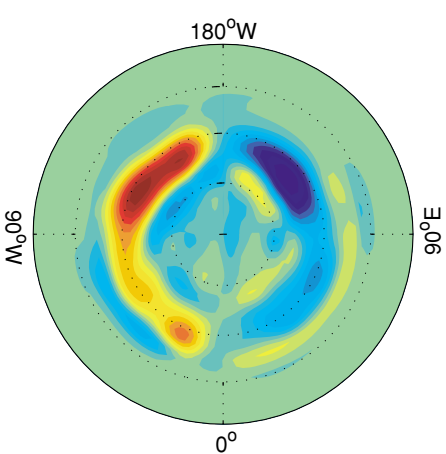

$\mathrm{J}_{\mathrm{r}}$ (grid) $\left[\mu \mathrm{A} / \mathrm{m}^{2}\right]$

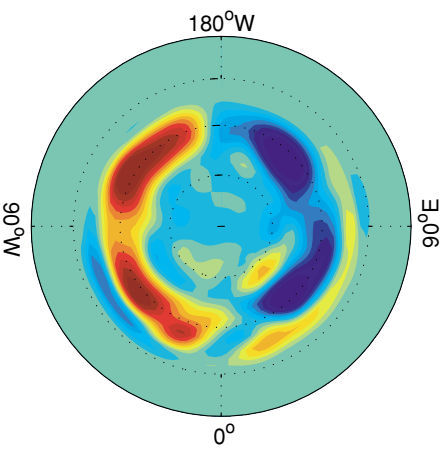

$J_{r}$ (grid) $\left[\mu A / m^{2}\right]$

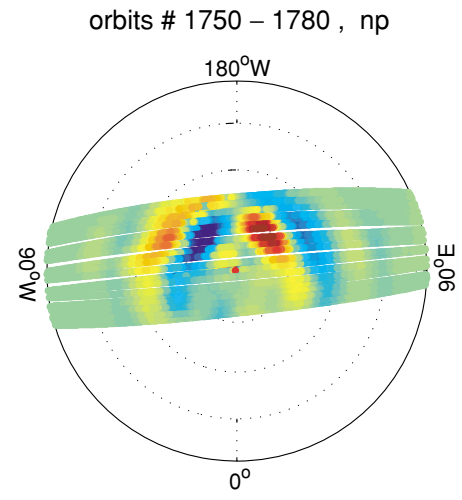

$\mathrm{J}_{\mathrm{z}}\left[\mu \mathrm{A} / \mathrm{m}^{2}\right]$

orbits \# 1750 - 1780 , np

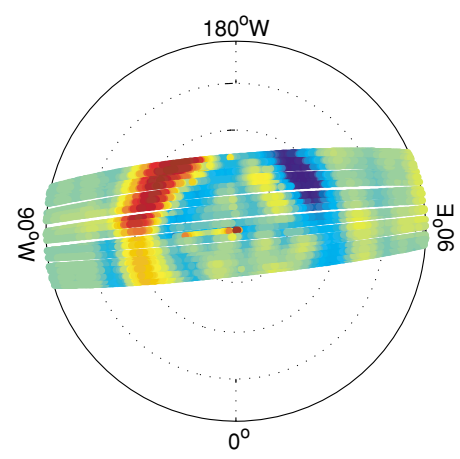

$\mathrm{J}_{\mathrm{z}}\left[\mu \mathrm{A} / \mathrm{m}^{2}\right]$

orbits \# $1750-1780$, np

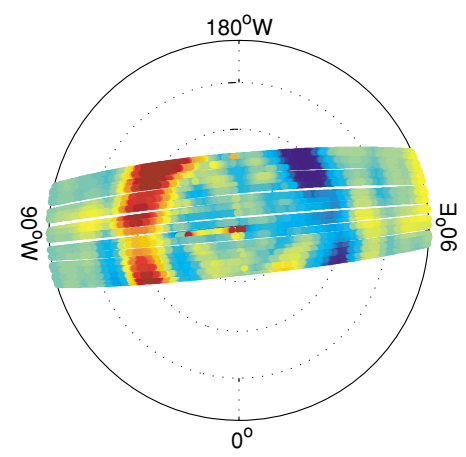

$J_{z}\left[\mu A / m^{2}\right]$

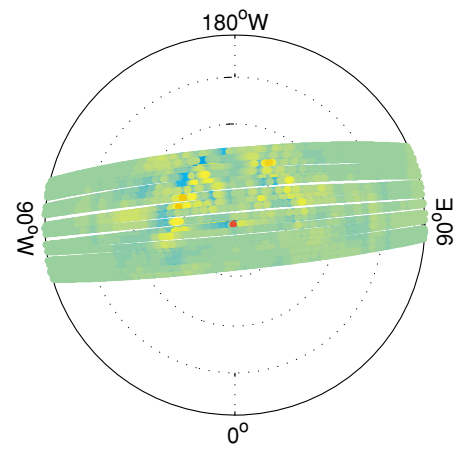

$$
J_{z}-J_{r}\left[\mu A / m^{2}\right]
$$

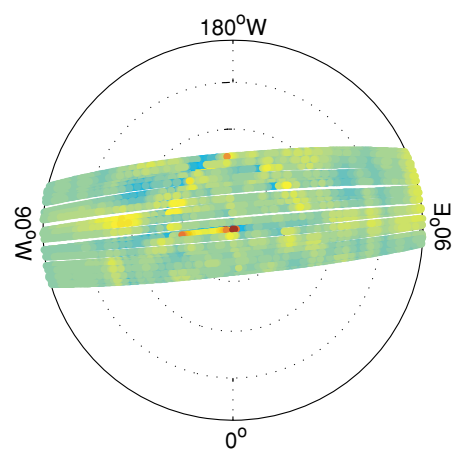

$$
J_{z}-J_{r}\left[\mu A / m^{2}\right]
$$

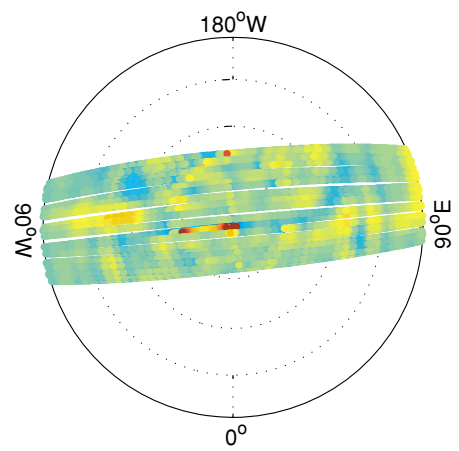

$J_{z}-J_{r}\left[\mu A / m^{2}\right]$

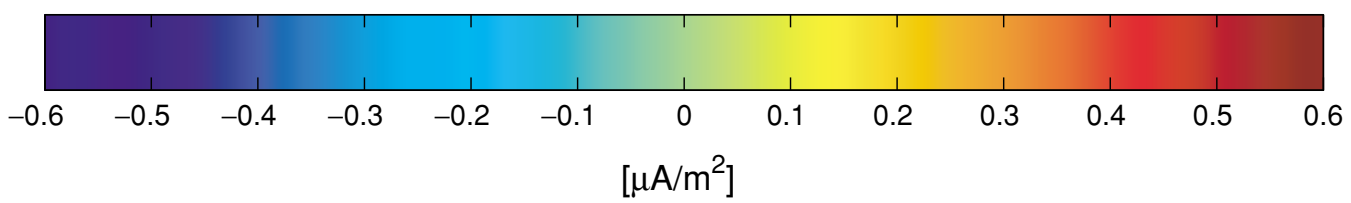

Fig. 5. Comparison of model radial currents with the derived vertical currents from orbits at 06:00 local time. (left column) distribution of radial currents from input model, (middle column) derived vertical currents from all orbits of a two-days period, (right panel) difference between derived and model currents. The rows represent different levels of activity, (top row) inter planetary magnetic field: $B_{Z}=5 \mathrm{nT}$, (middle row) $B_{Z}=-2.5 \mathrm{nT}$, (bottom row) $B_{Z}=-5 \mathrm{nT}$.

To get a better impression of the capability of the applied method for deriving field-aligned current patterns, a series of plots has been generated showing the modeled and recovered current distributions for 30 successive orbits on polar plots. Figures 5 and 6 show the radial current distribution at the north pole in Solar-Magnetic (SM) coordinates. Midnight is at $0^{\circ}$ and noon at $180^{\circ}$ longitude. The input currents are in the left column; the patterns recovered within a two-days period (assuming a static FAC distribution) are displayed in the middle, and the differences between the 


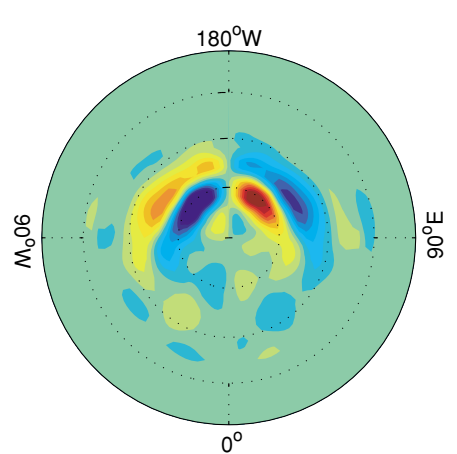

$J_{r}$ (grid) $\left[\mu \mathrm{A} / \mathrm{m}^{2}\right]$

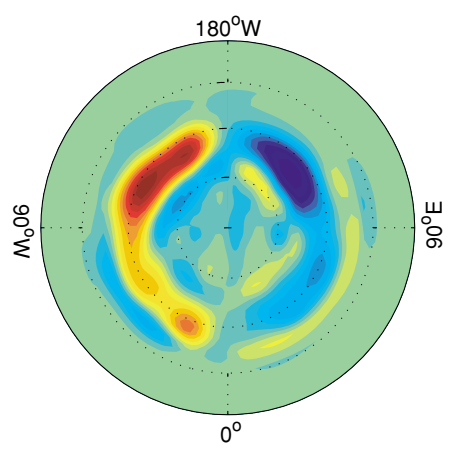

$J_{r}$ (grid) $\left[\mu \mathrm{A} / \mathrm{m}^{2}\right]$

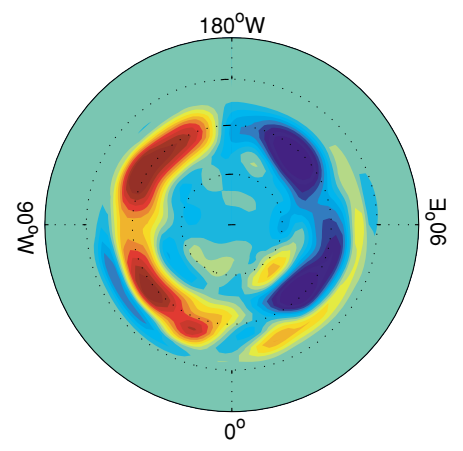

$J_{r}\left(\right.$ grid) $\left[\mu \mathrm{A} / \mathrm{m}^{2}\right]$

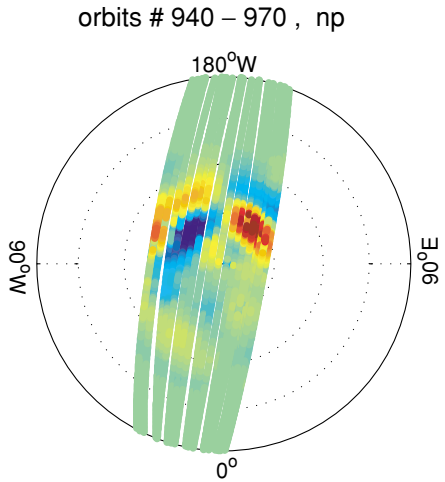

$\mathrm{J}_{\mathrm{z}}\left[\mu \mathrm{A} / \mathrm{m}^{2}\right]$

orbits \# $940-970, \mathrm{np}$

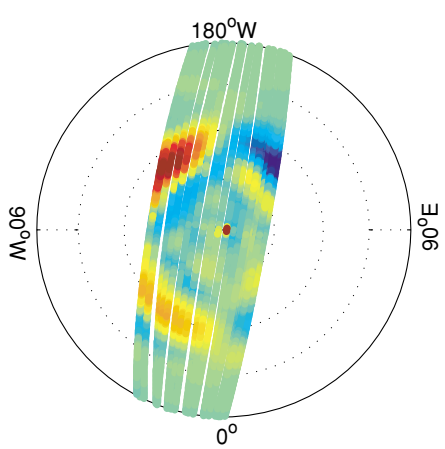

$\mathrm{J}_{\mathrm{z}}\left[\mu \mathrm{A} / \mathrm{m}^{2}\right]$

orbits \# $940-970, \mathrm{np}$

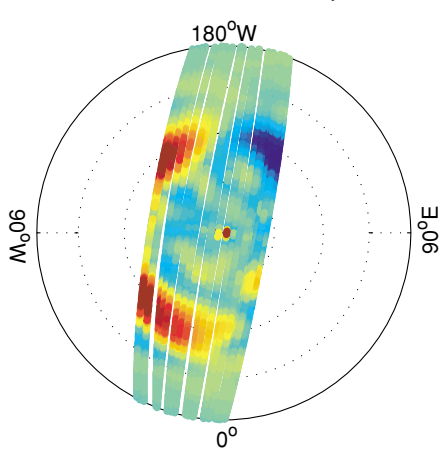

$\mathrm{J}_{\mathrm{z}}\left[\mu \mathrm{A} / \mathrm{m}^{2}\right]$

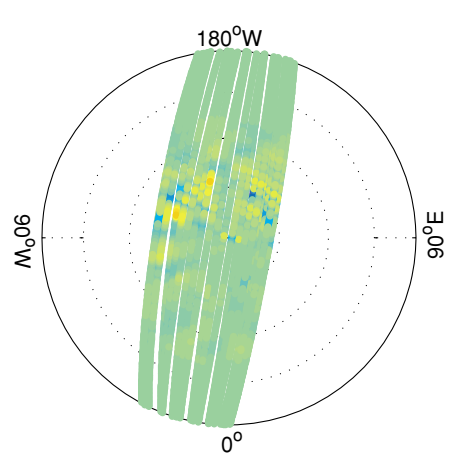

$J_{z}-J_{r}\left[\mu A / m^{2}\right]$

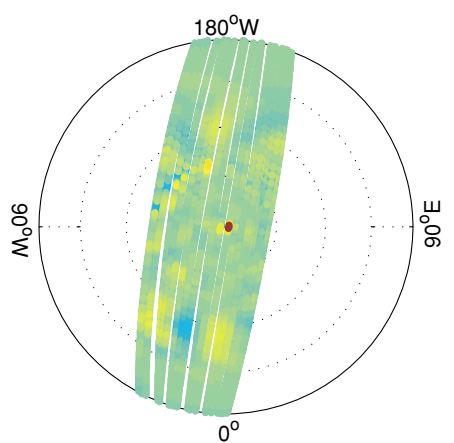

$J_{z}-J_{r}\left[\mu A / m^{2}\right]$

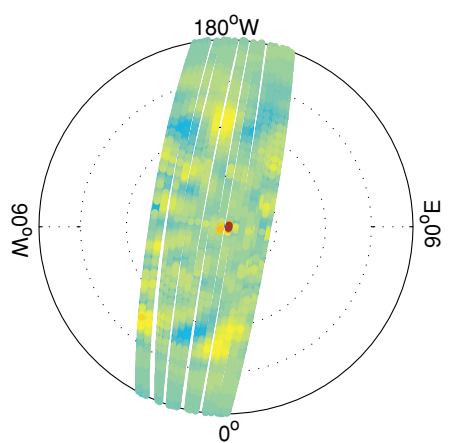

$J_{z}-J_{r}\left[\mu \mathrm{A} / \mathrm{m}^{2}\right]$

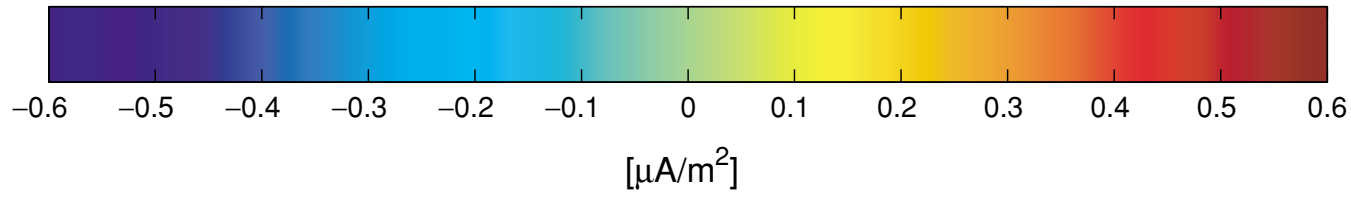

Fig. 6. Comparison of model radial currents with the derived vertical currents from orbits at 11:00 local time (format as in Fig. 5).

two are shown in the right column. From top to bottom, three scenarios with increasing levels of magnetic activity are considered. The MHD simulations for producing these data were run for IMF $B_{Z}$ conditions $5,-2.5$, and $-5 \mathrm{nT}$. In Fig. 5, the Swarm satellites are crossing the polar region from dawn to dusk. In Fig. 6 they move from noon to midnight. The purpose of this series of examples is to find out whether the orbit orientation with respect to the FAC sheets or the activity level has an influence on the quality of the obtained results. Due to the separation between the magnetic and geographic poles the satellites cover a swath of $2500 \mathrm{~km}$ width in the SM system every day.

When looking at the difference plots, some systematic features show up in the residuals. Right at the pole there is an isolated deflection. Especially in Fig. 5 a half circle of yellow dots are visible. They reflect the small phase shift. 
In all other cases no systematic relation can be identified.

\section{Comparison to Single Satellite Results}

Up to date, the estimates of ionospheric field-aligned currents have to rely on single satellite measurements. In that case the results depend on a number of assumptions. The most important one concerns the geometry of the currents (Lühr et al., 1996). Frequently, it is assumed that the spacecraft crosses a sheet of field-aligned currents at a right angle. In that case Eq. (3) can be reduced to

$$
j_{z}=\frac{1}{\mu_{0}} \frac{\partial B_{y}}{\partial x}
$$

Since the $x$ component of our VZ frame is aligned with the velocity vector, one may write

$$
j_{z}=\frac{1}{\mu_{0}} \frac{1}{v} \frac{\partial B_{y}}{\partial t}
$$

where $v$ is the orbital speed of the satellite.

As part of this Swarm study we tested also the reliability of the single satellite approach by using the same synthetic data set of auroral zone fields and currents. Magnetic field data sampled along the orbit of SwA were applied to Eq. (10). The calculations are performed in the local Cartesian VZ frame. The transformations into this frame are the same as described in the previous sections.

A comparison between the estimated radial current densities and the model data is shown in Fig. 7 for a selection of tracks of one day both for the north and south pole. For most crossings a reasonable agreement of the two curves can be observed. There are, however, some passes where the current density is badly underestimated (lower left and upper right). In this approach, too, we find a slight phase shift of the estimated current features towards the pole. We take this as a further indication that the shift is not caused by our curl-B method described above, but may rather be a mapping effect.

Figures 8 and 9 show the comparison of input and estimated current distribution on polar plots, as presented before in the multi-satellite approach. For the two local times of the orbit plane we get quite different results. As expected, the residuals are small in regions where the angle of attack between the flight direction and the current system is close to $90^{\circ}$. At the fringes of the swath, however, where this angle becomes smaller, the deficit in estimated current density is severe and reaches $50 \%$. The residuals of the dawn/dusk and the noon/midnight orbits look quite different now. From the comparison of the single satellite approach with the curl-B technique we may get an idea of the uncertainty involved in present-day field-aligned current estimates, if the orientation of the current sheet is not determined. This may be attempted e.g. by a minimum variance analysis. In case of low flying satellites $(<400 \mathrm{~km})$, however, this technique is significantly contaminated by magnetic signatures of horizontal currents.

\section{Discussion}

In this study we made use of the synthetic data set containing ionospheric currents and fields to develop and test methods for estimating ionospheric currents from Swarmtype observations at satellite altitude. The Swarm constellation is particularly well suited to determine field-aligned currents accurately as the mission will allow for the first time to use spatial differences of the magnetic field readings of two horizontally spaced satellites for this purpose.

The aim of this study is to judge the quality of the retrieved currents when using realistic Swarm orbit configurations. The current estimates are based on simulated measurements taken by the two lower satellites flying side-byside separated in east-west direction. Since the sampling geometry is changing continuously, the quality of the curlB method may also change.

The results presented in Section 3 are very promising. Reliable current densities can be obtained all along the orbit, as close as a few kilometres to the crossover point. The excellent agreement between input model and computed field-aligned currents confirms the viability of our approach.

From the comparison between the derived vertical currents and the associated model currents we find a small but systematic phase shift. The derived current features appear shifted poleward by about a fraction of one degree in latitude. We think that the reason for this phase shift could be the linear interpolation between the grid points when retrieving the samples along the orbits. The spacing of grid points is continuously changing when approaching the pole.

Large residuals show up around the closest approach to the pole. This is probably due to the singularity of the spherical components at the poles. The choice of a different coordinate system for the synthetic data may solve this problem. A suitable alternative would be geocentric coordinates. In that case, the immediate vicinity of the geographic poles, which are then singular points, will be left unsampled by the present Swarm orbit configuration, and the cross-over points coincide with the closest approaches to the poles. Omitting data in the vicinity of those points will enlarge the uncovered area at the poles only slightly, but would cure both problems. Testing these suggestions could be the topic of a follow-on study.

We have also tested Ampere's integral law (Eq. (7)). It turned out to be no improvement. The computational demands are higher for the transformation since the two horizontal components have to be rotated by different angles and are thus no longer orthogonal. For this reason we did not elaborate further on that technique.

For comparison, the same model data have been used in a single-satellite approach for estimating field-aligned currents. In many cases the obtained FAC densities compare very well with the model currents. As expected, the computed values fall short, up to $50 \%$ in some cases, when the angle of attack between the satellite velocity vector and the orientation of the FAC sheet is significantly smaller than $90^{\circ}$. This indicates the degree of uncertainty inherent in many present-day field-aligned current studies.

The other caveat concerning the single satellite approach lies in the influence of magnetic fields from other sources. The curl-B approach, as outlined in Section 1, rejects fields derived from scalar potentials. However, the reduced version, as given in Eq. (10), is sensitive to all field contri- 

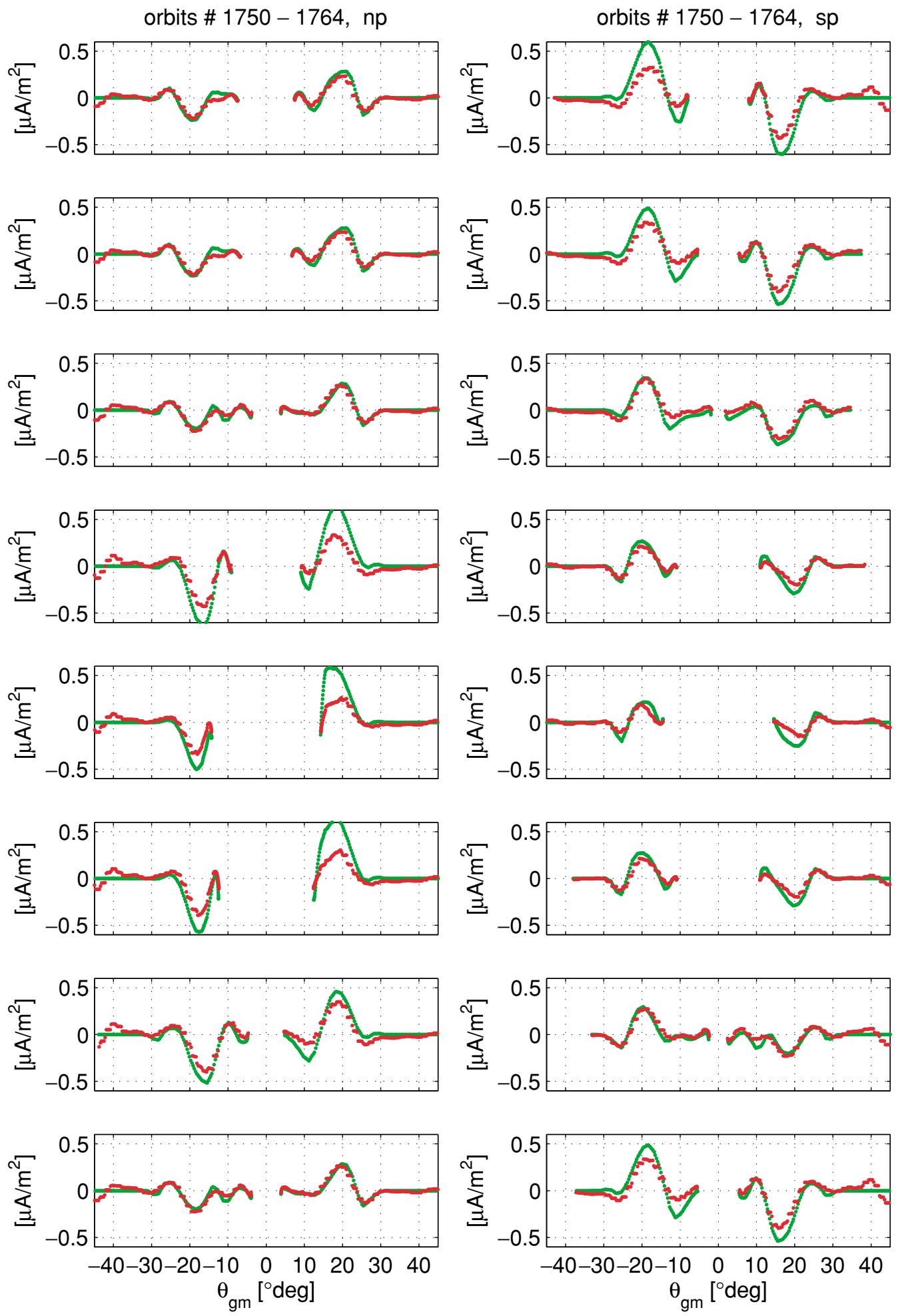

$$
\mathrm{j}_{\mathrm{z}} \mathrm{j}_{\mathrm{r}} \text { (grid) }
$$

Fig. 7. Direct comparison of derived radial current density using the single satellite (SwA) method (red) and the corresponding model input data (green) (IMF $B_{Z}=-2.5 \mathrm{nT}$ ).

butions. Thus, fields from horizontal E-region currents or crustal anomalies may cause spurious field-aligned current readings. This is not the case if the Swarm curl-B technique, as described in Eq. (5), is used. The importance of this aspect becomes increasingly relevant the lower a spacecraft flies.

Concerning the achievable resolution of the field-aligned current density, we may refer to the accuracy specified for the Swarm vector magnetic field. The accuracy is quoted to be $0.1 \mathrm{nT}$. When considering differences, $0.15 \mathrm{nT}$ can be 


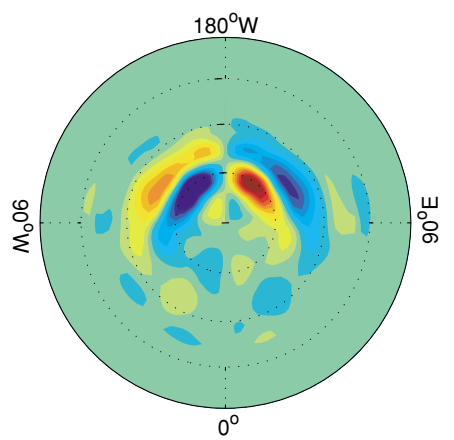

$J_{r}$ (grid) $\left[\mu \mathrm{A} / \mathrm{m}^{2}\right]$

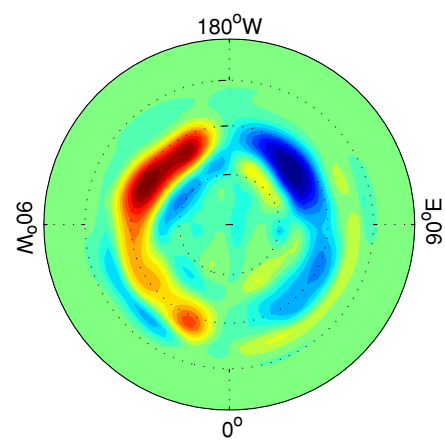

$J_{r}\left(\right.$ grid) $\left[\mu \mathrm{A} / \mathrm{m}^{2}\right]$

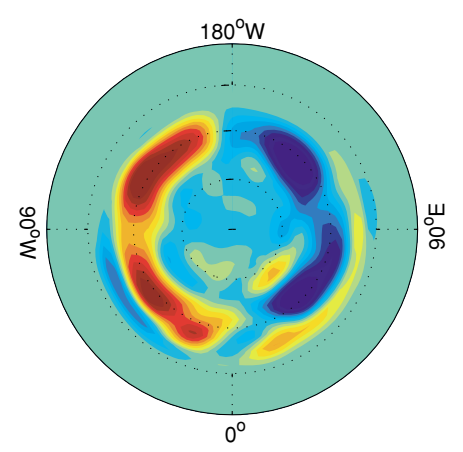

$J_{r}\left(\right.$ grid) $\left[\mu \mathrm{A} / \mathrm{m}^{2}\right]$

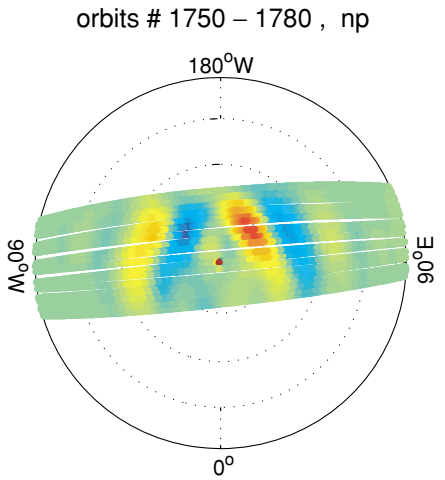

$$
\mathrm{J}_{\mathrm{z}}\left[\mu \mathrm{A} / \mathrm{m}^{2}\right]
$$

orbits \# $1750-1780$, np

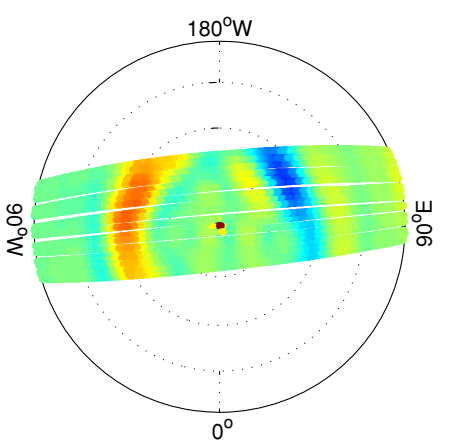

$\mathrm{J}_{\mathrm{z}}\left[\mu \mathrm{A} / \mathrm{m}^{2}\right]$

orbits \# $1750-1780$, np

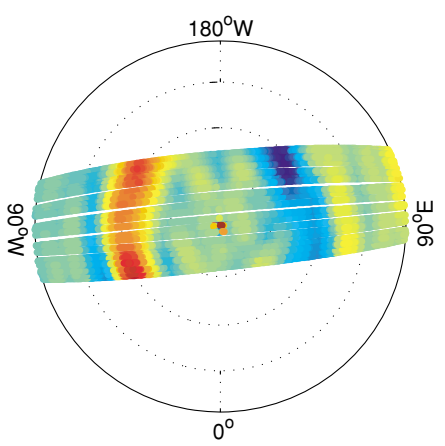

$\mathrm{J}_{\mathrm{z}}\left[\mu \mathrm{A} / \mathrm{m}^{2}\right]$

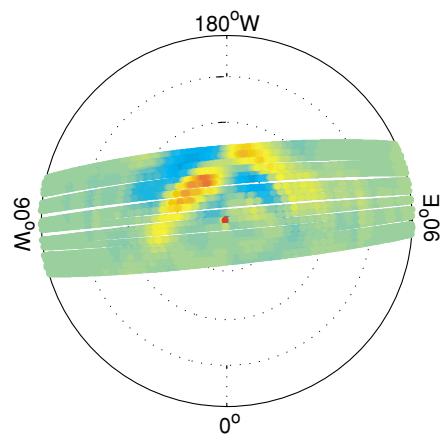

$J_{z}-J_{r}\left[\mu A / m^{2}\right]$

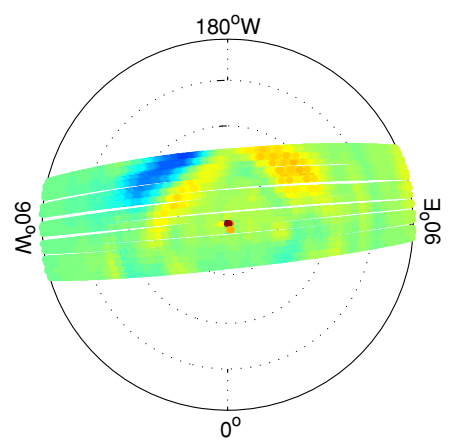

$$
J_{z}-J_{r}\left[\mu A / m^{2}\right]
$$

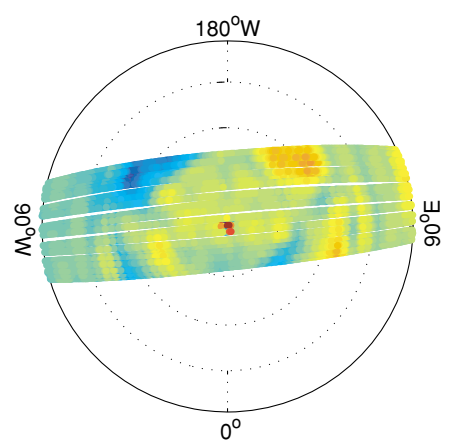

$J_{z}-J_{r}\left[\mu A / m^{2}\right]$

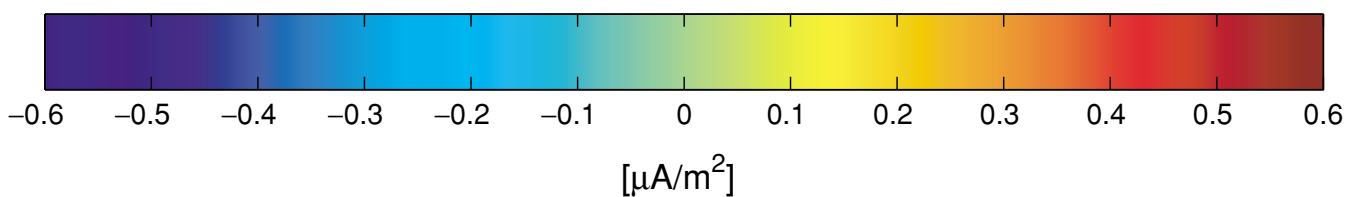

Fig. 8. Comparison of model radial currents with the single satellite (SwA) derived vertical currents from orbits at 06:00 local time. (left column) distribution of radial currents from input model, (middle column) derived vertical currents from all orbits of a two-days period, (right panel) difference between derived and model currents. The rows represent different levels of activity, (top row) inter planetary magnetic field: $B_{Z}=5 \mathrm{nT}$, (middle row) $B_{Z}=-2.5 \mathrm{nT}$, (bottom row) $B_{Z}=-5 \mathrm{nT}$.

assumed. Together with a typical spacecraft separation of $40 \mathrm{~km}$ we obtain

$$
j_{z}=\frac{1}{\mu_{0}} \sqrt{2 \cdot\left(\frac{0.15 \mathrm{nT}}{40 \mathrm{~km}}\right)^{2}}
$$

Thus, the resulting formal resolution of FAC density is $\Delta j_{z}= \pm 0.004 \mu \mathrm{A} / \mathrm{m}^{2}$, which is about $1 \%$ of a typical large-scale FAC amplitude.

So far we have applied the curl-B technique only to simulated data. As can be seen in Fig. 2 or Fig. 5, only the large-scale FACs are reproduced by the MHD models. In 


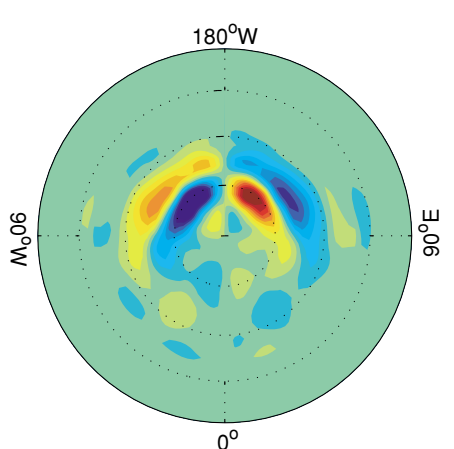

$J_{r}$ (grid) $\left[\mu \mathrm{A} / \mathrm{m}^{2}\right]$

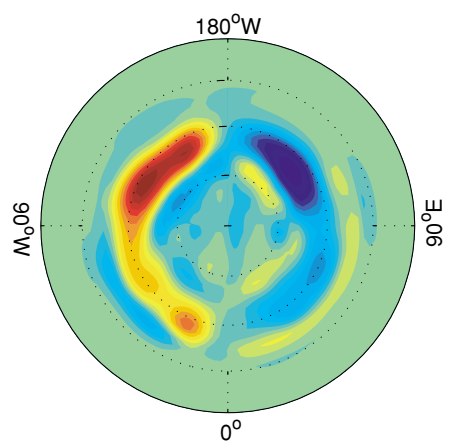

$J_{r}$ (grid) $\left[\mu \mathrm{A} / \mathrm{m}^{2}\right]$

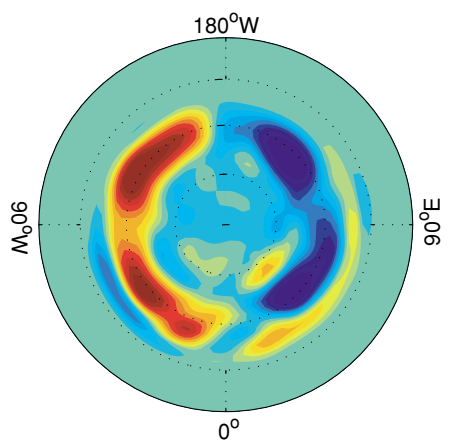

$J_{r}\left(\right.$ grid) $\left[\mu \mathrm{A} / \mathrm{m}^{2}\right]$

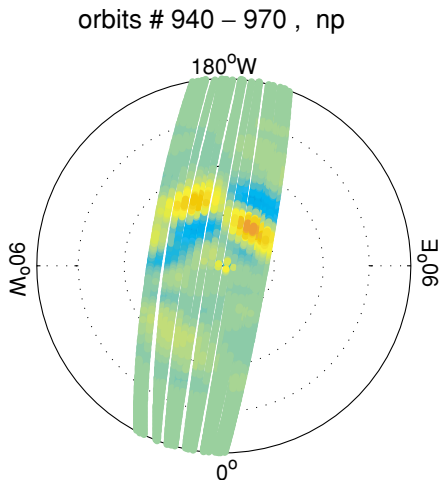

$\mathrm{J}_{\mathrm{z}}\left[\mu \mathrm{A} / \mathrm{m}^{2}\right]$

orbits \# $940-970, \mathrm{np}$

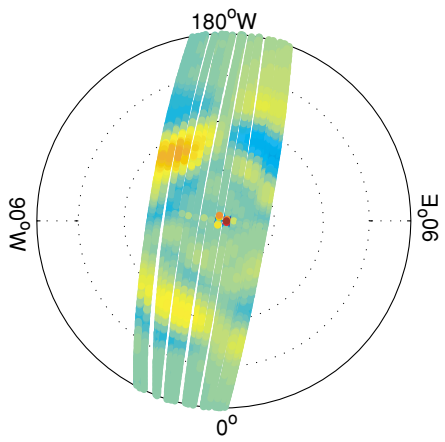

$\mathrm{J}_{\mathrm{z}}\left[\mu \mathrm{A} / \mathrm{m}^{2}\right]$

orbits \# $940-970, \mathrm{np}$

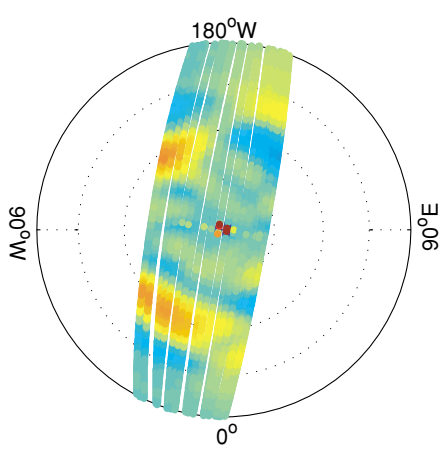

$\mathrm{J}_{\mathrm{z}}\left[\mu \mathrm{A} / \mathrm{m}^{2}\right]$

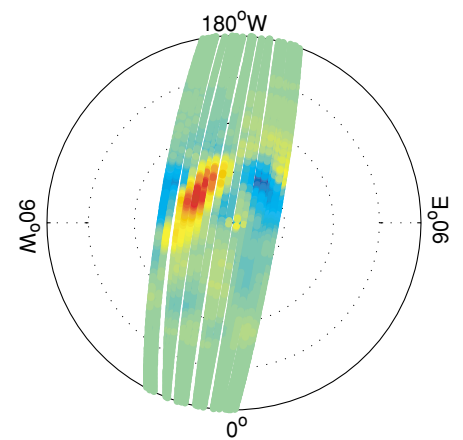

$J_{z}-J_{r}\left[\mu A / m^{2}\right]$

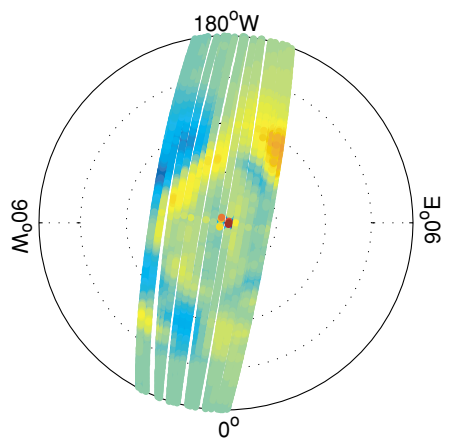

$J_{z}-J_{r}\left[\mu A / m^{2}\right]$

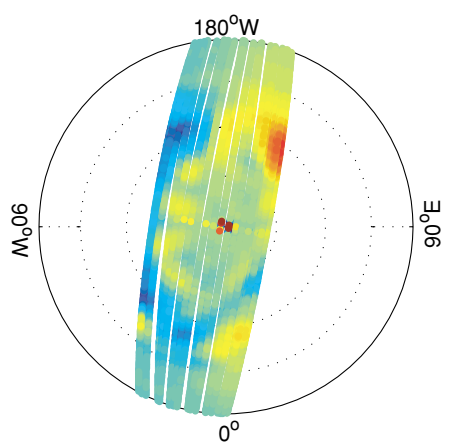

$\mathrm{J}_{z}-\mathrm{J}_{\mathrm{r}}\left[\mu \mathrm{A} / \mathrm{m}^{2}\right]$

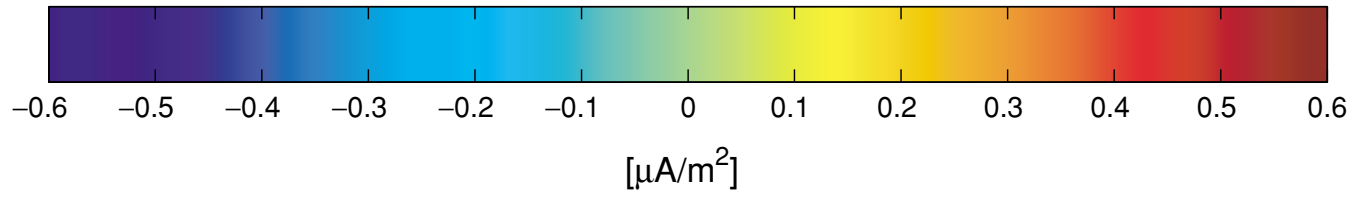

Fig. 9. Comparison of model radial currents with the derived vertical currents from orbits at 11:00 local time (format as in Fig. 8).

reality, FACs cover a wide range of spatial and temporal scales. The question is, which part of the spectrum is recovered properly by the Swarm curl-B technique? To answer this question, we take a look at actual observations.

Figure 10 shows FAC estimates derived from CHAMP measurements (Reigber et al., 2002) along a passage of the northern auroral region. First, the satellite crosses the oval in the pre-midnight local time sector, then in the pre-noon sector. In the top three frames, field-aligned current densities at different scales are shown. The general characteristic of FACs emerges, i.e. the smaller the spatial scale, the larger the current density. Peak current densities are attained by current filaments of about $1 \mathrm{~km}$ size, which are obtained from full resolution $50 \mathrm{~Hz}$ data (top panel). When averaging the data over $1 \mathrm{~s}$ (second panel), FACs with scales of a few tens of kilometers dominate, but their amplitudes are 
$2002 / 06 / 07$
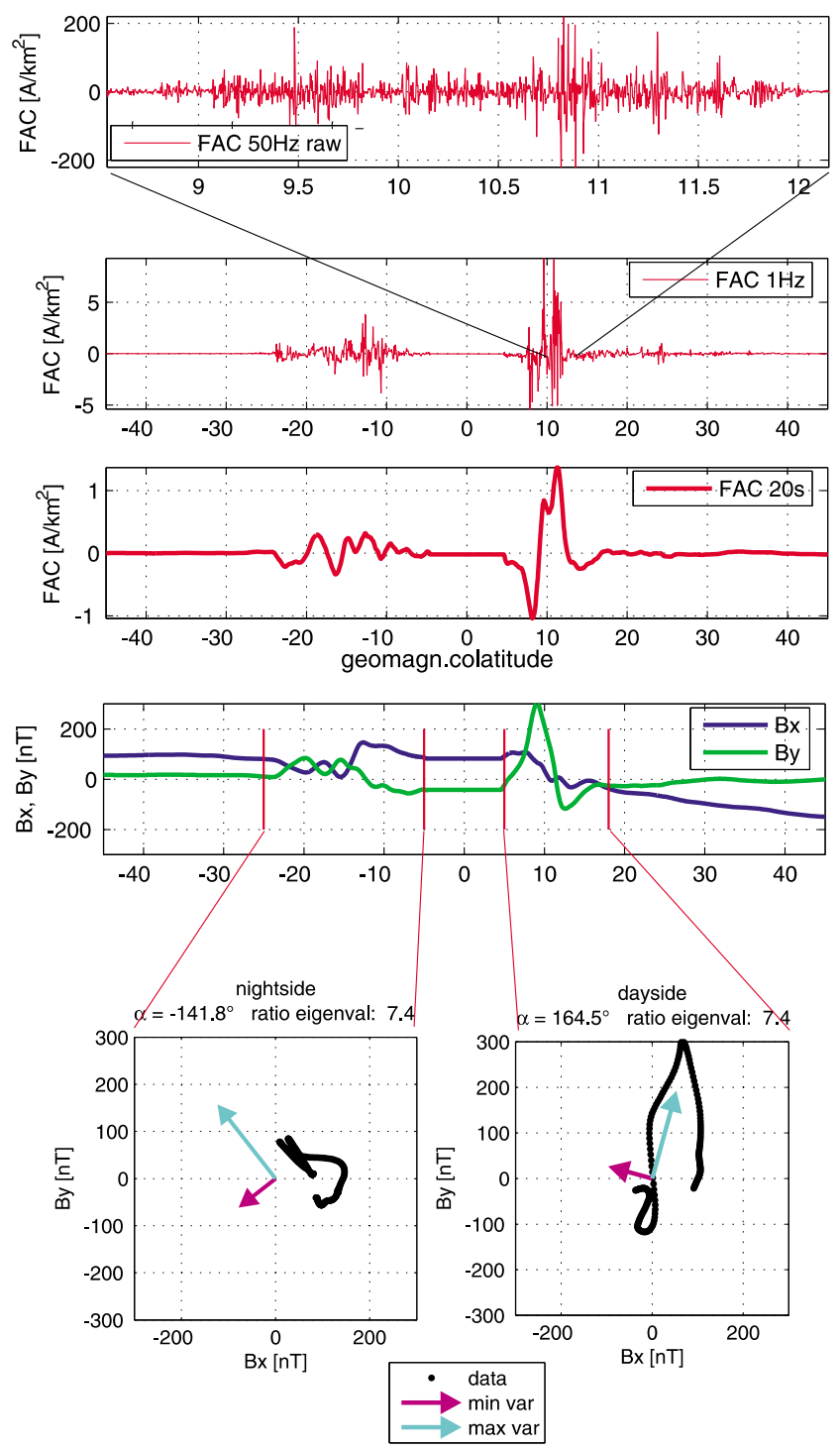

Fig. 10. FACs computed from CHAMP vector data. From top to bottom: $50 \mathrm{~Hz}$ FACs, $1 \mathrm{~s}$ FACs, $20 \mathrm{~s}$ FACs, horizontal components $B_{x}$ and $B_{y}$, and hodograms with directions of minimum/maximum variance for nightside and dayside sections, respectively.

already smaller by a factor of 30 compared to the fine scale FACs. Applying a $20 \mathrm{~s}$ low-pass filter (third panel) emphasizes the well-known large-scale FACs with wavelengths of several hundred kilometers. Their peak amplitudes are reduced by a further factor of 5 .

The presented curl-B technique can only respond properly to field-aligned currents that have scales significantly larger ( $\sim 4$ times) than the separation of the measurement points and that are constant during the time of passage (about $30 \mathrm{~s}$ ). When considering the measurement geometry as expected for the Swarm mission, we have to deal with cross-track separations of up to $100 \mathrm{~km}$ in the auroral region. In order to avoid aliasing, the magnetic field data need to be low-pass filtered before the curl-B technique is applied. The $20 \mathrm{~s}$ filter seems to be a suitable choice for that.

It is known that the large-scale FACs are often organized in elongated sheets aligned with the auroral oval. Since the Swarm satellites are going to pass the oval generally at a large angle it is justified to select a shorter sampling distance $(38 \mathrm{~km})$ for along-track than for cross-track calculations. We have tested the orientation of the current sheets of the pass presented in Fig. 10. The hodograms of the transverse magnetic fields (bottom frames) give a good impression. While the signature is nicely elongated at the dayside (right frame), the situation seems less clear on the nightside (left frame). This impression is not confirmed by the minimum variance analysis (MVA) (Sonnerup and Cahill, 1967). The ratio between the large and small eigenvalues is 7.4 for both, the day and nightside. The angle of attack between the orbit and the sheet as derived from the minimum variance is larger on the dayside $\left(74.5^{\circ}\right)$ than on the nightside $\left(51.8^{\circ}\right)$. These numbers indicate that the FACs on the nightside, for example, are underestimated by a factor of 0.8 by the single satellite method used here. It is not possible to decide how much the MVA is influenced by the electrojet. The foreseen Swarm constellation can cope with these problems and is well-suited to resolve the large-scale FAC distribution which results from low-pass filtered (20 s) magnetic field data.

In summary, testing the curl-B technique for the estimation of field-aligned currents with a synthetic dataset revealed excellent results. Remaining discrepancies are likely to be caused by the interpolation of the samples from the discrete synthetic data points. Due to the dimensions of spacecraft separations, only large-scale FACs can be recovered. They form the well-known local time pattern. Small and medium scale FACs are not accessible with the proposed constellation. As the formal resolution of the estimated FACs is very high (several $\mathrm{nA} / \mathrm{m}^{2}$ ), it seems possible to detect also the low-amplitude mid-latitude current signatures such as interhemispheric field-aligned currents (Olsen, 1997; Yamashita and Iyemori, 2003) with this dual-satellite technique.

Acknowledgments. We thank M. Rother for providing the CHAMP high resolution FAC data. ESA (ESTEC) supported this study through contract No. 3-10901/03/NL/CB.

\section{Appendix A. Coordinate Systems}

A number of different coordinate systems have been used in this study. Definitions and references are given within this section.

- Mean-Field-Aligned (MFA) Frame: The MFA is a local Cartesian system. The origin of the frame is the measurement point. The $z$ axis is aligned with the average magnetic field direction as defined by a suitable magnetic field model (e.g., IGRF or better) at the systems origin. The $y$ component is perpendicular to the local magnetic meridian, pointing eastward; the $x$ component completes the triad.

- Solar Magnetic (SM) Frame: The SM frame is in particular useful to describe the electrodynamics in the nearEarth space. In this study it is a spherical coordinate system-with the components $(r, \theta, \phi)$ defined by the orientation of the geomagnetic dipole. The origin of the frame is the centre of the Earth. The origin of the colatitude, $\theta$, is the geomagnetic dipole axis at the northern pole. The origin 
of the longitude, $\phi$, is defined by the plane containing the dipole axis and the direction to the sun. The $\phi=0^{\circ}$-line is on the night-side and values increase towards east.

- IERS Conventional Terrestrial Reference Frame (ITRF): The ITRF is an Earth-fixed Cartesian system used in this study for describing the orbit ephemerides. The origin of the frame is the Earth's centre of mass. The $x$ axis points towards the IERS Reference Meridian (close to Greenwich); the $z$-axis points to the Reference North Pole; the $y$-axis completes the triad. A detailed description of this frame can be found in the IERS Technical Note 21 (McCarthy, 1996).

- Velocity Zenith (VZ) Frame: The Velocity-Zenith frame is a locally horizontal Cartesian system. The origin is the center of the four measurement points. The $z$ axis points radially outward; the $x$ axis lies in the plane containing the $z$ axis and the mean velocity vector of the two spacecraft, pointing in flight direction; the $y$ axis completes the triad laying in the horizontal plane.

\section{References}

Anderson, B. J., K. Takahashi, and B. A. Toth, Sensing global Birkeland currents with Iridium engineering magnetometer data, Geophys. Res. Lett., 27, 4045, 2000.

Friis-Christensen, E., H. Lühr, and G. Hulot, Swarm: A constellation to study the Earth's magnetic field, Earth Planets Space, 58, this issue, 351-358, 2006.

Fung, S. F. and R. A. Hoffman, Finite geometry effects of field-aligned currents, J. Geophys. Res., 97, 8569-8579, 1992.

Holme, R., N. Olsen, M. Rother, and H. Lühr, CO2-a CHAMP magnetic field model, in "First CHAMP Mission Results for Gravity, Magnetic and Atmospheric Studies”, edited by C. Reigber, H. Lühr, and P. Schwintzer, pp. 220-225, Springer, Berlin, 2003.

Iijima, T. and T. Potemra, The amplitude distribution of field-aligned currents at northern high latitudes observed by TRIAD, J. Geophys. Res., 81, 2165-2174, 1976.

Lühr, H., J. J. Warnecke, and M. Rother, An algorithm for estimating fieldaligned currents from single spacecraft magnetic field measurements: a diagnostic tool applied to Freja satellite data, IEEE Trans. Geosci. Remote Sens., 34, 1369-1376, 1996.

McCarthy, D., IERS Conventions (1996), IERS Technical Note 21, Observatoire de Paris, July, 1996.

Moretto, T., S. Vennerstrøm, N. Olsen, L. Rastätter, and J. Raeder, Using global magnetospheric models for simulation and interpretation of Swarm external field measurements, Earth Planets Space, 58, this issue,
439-449, 2006.

Olsen, N., Ionospheric F region currents at middle and low latitudes estimated from MAGSAT data, J. Geophys. Res., 102(A3), 4563-4576, 1997.

Olsen, N., et al., Swarm End-to-End Mission Performance Simulator Study, February 2004, ESA Contract No. 17263/03/NL/CB, DSRI Report 1/2004, 2004.

Reigber, C., H. Lühr, and P. Schwintzer, CHAMP mission status, Adv. Space Res., 30(2), 129-134, 2002.

Sonnerup, B. U. O. and L. J. Cahill, Magnetopause structure and attitude from explorer 12 observations, J. Geophys. Res., 72, 171-183, 1967.

Stauning, P., Field-aligned ionospheric current systems observed from the Magsat and Ørsted satellites during northward IMF, Geophys. Res. Lett., 29, 10.1029/2001GL013,961, 2002.

Stauning, P., F. Primdahl, J. Watermann, and O. Rasmussen, IMF Byrelated cusp currents observed from the Ørsted satellite and from ground, Geophys. Res. Lett., 28, 99-102, 2001.

Stauning, P., F. Christiansen, and J. Watermann, On the modelling of fieldaligned currents from magnetic observations by polar orbiting satellites, in Earth Observation with CHAMP, edited by C. Reigber, H. Lühr, P. Schwintzer, and J. Wickert, p. 371, Springer, Berlin, 2005.

Vennerstrøm, S., T. Moretto, N. Olsen, E. Friis-Christensen, A. Stampe, and J. Watermann, Field-aligned currents in the dayside cusp and polar cap region during northward IMF, J. Geophys. Res., 107(A8), 10.1029/2001JA009,162, 2002.

Vennerstrøm, S., E. Friis-Christensen, H. Lühr, T. Moretto, N. Olsen, C. Manoj, P. Ritter, L. Rastätter, A. Kuvshinov, and S. Maus, The impact of combined magnetc and electric field analysis and of ocean circulation effects on Swarm Mission performance, ESA Contract No. 310901/03/NL/CB, DSRI Report 2/2004, 2004.

Vennerstrom, S., T. Moretto, L. Rastätter, and J. Raeder, Modeling and analysis of solar wind generated contributions to the near-Earth magnetic field, Earth Planets Space, 57, this issue, 451-461, 2006.

Waters, C. L., B. J. Anderson, and K. Liou, Estimation of global fieldaligned currents using the Iridium System magnetometer data, Geophys. Res. Lett, 28(11), 2165-2168, 2001.

Yamashita, S. and T. Iyemori, Seasonal and local-time dependences of the inter-hemispheric field-aligned currents deduced from the Ørsted satellite and the ground geomagnetic observations, in OIST-4 Proceedings, edited by P. Stauning, H. Lühr, P. Ultré-Guérard, J. LaBreque, M. Purucker, F. Primdahl, J. Joergenson, F. Christiansen, P. Hoeg, and K. Lauritsen, p. 159, DMI, Copenhagen, 2003.

Zmuda, A. J. and J. C. Armstrong, The diurnal flow pattern of field-aligned currents, J. Geophys. Res., 79, 4611-4619, 1974.

Zmuda, A. J., J. H. Martin, and F. T. Heuring, Transverse magnetic disturbances at 1100 kilometers in the auroral region, J. Geophys. Res., 71, 5033-5045, 1966.

P. Ritter (e-mail: pritter@gfz-potsdam.de) and H. Lühr 